\title{
A Least Squares Interpretation of Sub-Space Methods for System Identification. ${ }^{1}$
}

\author{
Lennart Ljung and Tomas McKelvey \\ Dept. of Electrical Engineering, Linköping University \\ S-581 83 Linköping, Sweden, \\ Email: ljung@isy.liu.se, tomas@isy.liu.se.
}

CDC 1996, Kobe Japan

\begin{abstract}
So called subspace methods for direct identification of linear models in state space form have drawn considerable interest recently. The algorithms consist of series of quite complex projections, and it is not so easy to intuitively understand how they work. They have also defied, so far, complete asymptotic analysis of their stochastic properties. This contribution describes an interpretation of how they work. It specifically deals how consistent estimates of the dynamics can be achieved, even though correct predictors are not used. We stress how the basic idea is to focus on the estimation of the state-variable candidates - the $k$-step ahead output predictors.
\end{abstract}

\section{Introduction}

A linear system can always be represented in state space form as

$$
\begin{aligned}
x(t+1) & =A x(t)+B u(t)+w(t) \\
y(t) & =C x(t)+D u(t)+\nu(t)
\end{aligned}
$$

We shall generally let $n$ denote the dimension of $x$ and let $p$ be the number of outputs. To estimate such a model, the matrices can be parameterized either from physical grounds or as black boxes in canonical forms. Then these parameters can be estimated using prediction error/maximum likelihood (ML) techniques. See, e.g. [5].

However, there are also other possibilities: So called subspace methods, [9], [10], [2], [12], [13] form an interesting alternative to the ML approach. The idea behind these methods can be explained as first estimating the state vector $x(t)$, and then finding the state space matrices by a linear least squares procedure. These meth-

\footnotetext{
${ }^{1}$ This work was supported in part by the Swedish Research Council for Engineering Sciences (TFR), which is gratefully acknowledged.
}

ods are most often described in a geometric framework, which gives nice projection interpretations.

We shall in this contribution describe the subspace approach in a conventional least squares estimation framework. This give some complementary insight, which could be useful for development of alternative algorithms and for the asymptotic analysis.

\section{The Basic Idea}

Let us for a moment assume that not only are $u$ and $y$ measured, but also the sequence of state vectors $x$. This would, by the way, fix the state-space realization coordinate basis. Now, with known $u, y$ and $x$, the model (1) becomes a linear regression: the unknown parameters, all of the matrix entries in all the matrices, mix with measured signals in linear combinations. To see this clearly, let

$$
\begin{aligned}
& Y(t)=\left(\begin{array}{c}
x(t+1) \\
y(t)
\end{array}\right), \quad \Theta=\left(\begin{array}{ll}
A & B \\
C & D
\end{array}\right) \\
& \Phi(t)=\left(\begin{array}{c}
x(t) \\
u(t)
\end{array}\right), \quad E(t)=\left(\begin{array}{c}
w(t) \\
\nu(t)
\end{array}\right)
\end{aligned}
$$

Then, (1) can be rewritten as

$$
Y(t)=\Theta \Phi(t)+E(t)
$$

From this all the matrix elements in $\Theta$ can be estimated by the simple least squares method. The covariance matrix for $E(t)$ can also be estimated easily as the sample sum of the squared model residuals. That will give the covariance matrices for $w$ and $\nu$, as well as the cross covariance matrix. These matrices will, among other things, allow us to compute the Kalman filter for (1). Note that all of the above holds without changes for multivariable systems, i.e., when the output and input signals are vectors.

The problem is where to get the state vector sequence $x$ from. For that we turn to basic realization theory, as 
developed by [3], [1] and [7]. (See Appendix 4.A in [5] for an account). The basic results are as follows (see Lemmas 4A.1 and 4A.2 in [5] and their proofs):

Let a system be given by the impulse response representation

$$
y(t)=\sum_{j=0}^{\infty}\left(h_{u}(j) u(t-j)+h_{e}(j) e(t-j)\right)
$$

where $u$ is the input and $e$ the innovations. Let the $k$-step ahead predictors be defined by

$$
\hat{y}(t \mid t-k)=\sum_{j=k}^{\infty}\left(h_{u}(j) u(t-j)+h_{e}(j) e(t-j)\right)
$$

Notice, and this is important, that the input between time $t-k$ and $t$ are ignored: no attempt to predict its values from past data is made. Define

$$
\hat{Y}_{r}(t)=\left(\begin{array}{c}
\hat{y}(t \mid t-1) \\
\vdots \\
\hat{y}(t+r-1 \mid t-1)
\end{array}\right)
$$

Then the following is true:

1. The system (3) admits an $n$-th order state space description if and only if the rank of $\hat{Y}_{r}(t)$ is $\leq n$ for all $r$.

2. If rank $\hat{Y}_{n+1}=n$ then $n$ is the order of a minimal realization.

3. The state vector of any such minimal realization can be chosen as linear combinations of $\hat{Y}_{n}$ that form a basis for $\hat{Y}_{r}, r \geq n$, i.e.,

$$
x(t)=L \hat{Y}_{n}(t)
$$

such that $x(t)$ spans also $\hat{Y}_{n+1}(t)$.

Remark: "Rank", "basis" and "span" refer to the matrix obtained by the sequence of vectors $\mathcal{Y}_{N}=$ $\left[\hat{Y}_{n}(1), \hat{Y}_{n}(2), \ldots, \hat{Y}_{n}(N)\right]$

Note that the common canonical state space representations correspond to $L$ matrices that just pick out certain rows of $\hat{Y}_{n}$. In general, we are not confined to such choices, but may pick $L$ so that $x(t)$ becomes a well conditioned basis.

It is clear that the facts above will allow us to find a suitable state vector from data. The only remaining problem is to estimate the $k$-step ahead predictors. The true predictor $\hat{y}(t+k \mid t)$ is given by (4) and is a linear function of $u(i), y(i), \quad i \leq t$. For practical reasons the predictor is approximated so that it only depends on $s$ past data, $t-s+1 \leq i \leq t$. It can then efficiently be determined by another linear least squares projection directly on the input output data. That is, set up the model

$y(t+k)=\left(\theta^{k, \ell, s}\right)^{T} \varphi_{s}(t+1)+\left(\gamma^{k, \ell, s}\right)^{T} \tilde{\varphi}_{\ell}(t+1)+\epsilon(t+k)$

where

$$
\begin{gathered}
\varphi_{s}(t+1)=[y(t), u(t), \ldots, y(t-s+1), u(t-s+1)]^{T} \\
\tilde{\varphi}_{\ell}(t+1)=[u(t+1), \ldots, u(t+\ell)]^{T}
\end{gathered}
$$

Estimate $\theta$ and $\gamma$ in (7) using least squares giving $\hat{\theta}_{N}^{k, \ell, s}$ and $\hat{\gamma}_{N}^{k, \ell, s}$. The $k$-step ahead predictor is then

$$
\hat{y}_{s, \ell}(t+k \mid t)=\left(\hat{\theta}_{N}^{k, \ell, s}\right)^{T} \varphi_{s}(t+1)
$$

For large enough $s$ this will give a good approximation of the true predictors. Let us also introduce the predictor that includes future $u$ :

$$
\bar{y}_{s, \ell}(t+k \mid t)=\left(\hat{\theta}_{N}^{k, \ell, s}\right)^{T} \varphi_{s}(t+1)+\left(\hat{\gamma}_{N}^{k, \ell, s}\right)^{T} \tilde{\varphi}_{\ell}(t+1)
$$

Remark: The complication with the term $\gamma$ has the following reason: The values of $u(t+1), \ldots, u(t+k)$ affect $y(t+k)$, but should not be included in the predictor as demanded by (4). If $u$ is not white noise, future inputs can be predicted from past ones. Without the term $\gamma$ in (7) the first term would attempt to include the (predicted) effects from $\tilde{\varphi}(t+1)$ on $y(t+k)$, thus giving the wrong result.

The method thus consists of the following steps:

\section{Basic Subspace Algorithm}

1. Estimate $\hat{y}_{s, \ell}(t+k \mid t), k=1, \ldots, r$ using (10).

2. Form $\hat{Y}_{r}(t)$ in $(5)$

3. Estimate its rank and determine $L$ in (6)

4. Estimate $A, B, C, D$ and the noise covariance matrices using (2)

What we have described now is the subspace projection approach to estimating the matrices of the state-space model (1), including the basis for the representation and the noise covariance matrices. There are a number of variants of this approach. See among several references, e.g. [10], [2].

The approach gives very useful algorithms for model estimation, and is particularly well suited for multivariable systems. The algorithms also allow numerically very reliable implementations. They contain a number of choices and options, like how to choose $\ell, s$ and $r$, 
and also how to carry out step number 3 . There are also several "tricks" to do step 4 so as to achieve consistent estimates even for finite values of $s$. Accordingly, several variants of this method exist. In the following sections we shall give more algorithmic details around this approach.

\section{Efficient calculation of $\hat{Y}_{r}(t)$}

Let us now consider in somewhat more detail the subspace algorithm (12). In fact, there are many variants of these algorithms and for a comprehensive treatment, we refer to [11]. Here we shall only point to the essential features, and what elements account for the consistency. Recall the basic estimates (7)-(11). The corresponding vectors of stacked predictors will be denoted by

$$
\hat{Y}_{r}^{s, \ell}(t+1)=\left(\begin{array}{c}
\hat{y}_{s, \ell}(t+1 \mid t) \\
\vdots \\
\hat{y}_{s, \ell}(t+r \mid t)
\end{array}\right)
$$

and $\bar{Y}_{r}^{s, \ell}(t+1)$ analogously Clearly, we can treat all predictors simultaneously: Let

$$
Y_{r}(t+1)=\left(\begin{array}{c}
y(t+1) \\
\vdots \\
y(t+r)
\end{array}\right)
$$

and stack $r$ equations like (7) on top of each other:

$Y_{r}(t+1)=\Theta^{r, \ell, s} \varphi_{s}(t+1)+\Gamma^{r, \ell, s} \tilde{\varphi}_{\ell}(t+1)+E(t+1)$

Estimate the $p r \times s(p+m)$ matrix $\Theta($ together with $\Gamma)$ by least squares and then form

$$
\begin{aligned}
& \hat{Y}_{r}^{s, \ell}(t+1)=\hat{\Theta}_{N}^{r, \ell, s} \varphi_{s}(t+1) \\
& \bar{Y}_{r}^{s, \ell}(t+1)=\hat{\Theta}_{N}^{r, \ell, s} \varphi_{s}(t+1)+\hat{\Gamma}_{N}^{r, \ell, s} \tilde{\varphi}_{\ell}(t+1)
\end{aligned}
$$

In fact, these quantities can be efficiently calculated by projections using the data vectors, without explicitly forming the matrices $\hat{\Theta}$ and $\hat{\Gamma}$.

\section{Choice of order and basis}

We now have the (pr-dimensional) vector $\hat{Y}_{r}(t)$ for $t=1, \ldots, N$. If the system is of order $n$, this vector sequence has rank $n$, and we should select a basis for it by forming linear combinations

$$
x(t)=L \hat{Y}_{r}(t)
$$

so that $x$ becomes well conditioned. To find the rank of $\left\{\hat{Y}_{r}(t), t=1, \ldots, N\right\}$ we would form the $(p r \times N)$ matrix

$$
\mathcal{Y}_{N}=\left[\hat{Y}_{r}(1) \ldots \hat{Y}_{r}(N)\right]
$$

We could then perform an SVD on $\mathcal{Y}_{N}$ :

$$
\mathcal{Y}_{N}=U S V^{T}
$$

For added flexibility and options, the SVD could be carried out on a weighted version of $\mathcal{Y}_{N}$ :

$$
W_{1} \mathcal{Y}_{N} W_{2}=U S V^{T}
$$

(Here $W_{1}$ is a $p r \times p r$ matrix, while $W_{2}$ is $N \times N$.) In the sequel, we will not use these weighting matrices, though. We would now examine the singular values in $S$ and put those below a certain threshold to zero. Denote the number of singular values above the threshold by $n$. Let $S_{1}$ be the upper $n \times n$ part of $S$. The corresponding $n$ columns of $U$ will be denoted by $U_{1}$ (thus a $p r \times n$ matrix), and the corresponding columns of $V$ are $V_{1}$ so that

$$
U S V^{T} \approx U_{1} S_{1} V_{1}^{T}
$$

There are now several candidates for matrices $L$ in (18). The most common choice seems to be

$$
L=\left(U_{1} S_{1}^{1 / 2}\right)^{\dagger}
$$

\section{Relationships for the true predictors}

Suppose the true system can be described as (3), with its $k$-step ahead predictor given by (4). An immediate consequence is that

$$
\hat{y}(t+k \mid t)=\hat{y}(t+k \mid t-1)+h_{u}(k) u(t)+h_{e}(k) e(t)
$$

Suppose now that the true system can be written as a difference equation

$$
A_{0}(q) y(t)=B_{0}(q) u(t)+C_{0}(q) e(t)
$$

where the polynomials in the shift operator are all of degree at most $n$ :

$$
A_{0}(q)=I+A_{1} q^{-1}+\ldots A_{n} q^{-n}
$$

Then

$$
\hat{y}(t+r \mid t)+A_{1} \hat{y}(t+r-1 \mid t)+\ldots A_{n} \hat{y}(t+r-n \mid t)=0
$$

for any $r>n$. The proof is immediate: Take equation (25) with $t=t+r$ and project it onto the space spanned by $\{e(s), u(s), s \leq t\}$ (ignoring that future $u$ might be predicted from past). The left hand side equals the left hand side of (27), while the right hand side is zero if $r>n$.

Let is now confine the discussion to the single output case. (It can be exactly transferred to the multi-output case, at the expense of somewhat more complex expression.) 
Now, look at $\hat{Y}_{r}(t)$ made up of the true predictors. Equation (27) means that any row $k>n$ can be written as a linear combination of the rows above it. The rank of $\hat{Y}_{r}(t)$ is thus at most $n$, and a possible basis is formed by the $n$ first rows. Let us first choose an $L$ that picks these rows:

$$
x(t)=L \hat{Y}_{r}(t)=\hat{Y}_{n}(t)
$$

For component $k$ of this state vector we thus have

$$
\begin{aligned}
x_{k}(t+1)= & \hat{y}(t+k \mid t) \\
= & \hat{y}(t+k \mid t-1)+h_{u}(k) u(t)+h_{e}(k) e(t) \\
= & x_{k+1}(t)+h_{u}(k) u(t)+h_{e}(k) e(t) \\
x_{n}(t+1)= & \hat{y}(t+n \mid t) \\
= & \hat{y}(t+n \mid t-1)+h_{u}(n) u(t)+h_{e}(n) e(t) \\
= & -a_{1} \hat{y}(t+n-1 \mid t-1)-\ldots-a_{n} \hat{y}(t \mid t-1) \\
& +h_{u}(n) u(t)+h_{e}(n) e(t) \\
= & -a_{1} x_{n}(t)-\ldots-a_{n} x_{1}(t)+h_{u}(n) u(t) \\
& +h_{e}(n) e(t)
\end{aligned}
$$

using (24) and (27). In matrix notation we have

$$
\begin{gathered}
x(t+1)=A_{c} x(t)+\left(\begin{array}{c}
h_{u}(1) \\
h_{u}(2) \\
\vdots \\
h_{u}(n)
\end{array}\right) u(t)+\left(\begin{array}{c}
h_{e}(1) \\
h_{e}(2) \\
\vdots \\
h_{e}(n)
\end{array}\right) e(t) \\
y(t)=C_{c} x(t)+h_{u}(0) u(t)+h_{e}(0) e(t)
\end{gathered}
$$

where

$$
\begin{gathered}
A_{c}=\left(\begin{array}{ccccc}
0 & 1 & 0 & \ldots & 0 \\
0 & 0 & 1 & \ldots & 0 \\
\vdots & \vdots & \vdots & \vdots & \\
0 & 0 & 0 & \ldots & 1 \\
-a_{n} & -a_{n-1} & -a_{n-2} & \ldots & -a_{1}
\end{array}\right) \\
C_{c}=\left(\begin{array}{lllll}
1 & 0 & 0 & \ldots & 0
\end{array}\right)
\end{gathered}
$$

This is of course the standard observability canonical form. See [4].

Suppose now that we pick another $L$ in (28). Let us first note that, since the first $n$ rows form a basis, we can always write

$$
\hat{Y}_{r}(t)=F \hat{Y}_{n}(t)=F x(t)
$$

for some $p r \times n$ matrix $F$. Choosing an arbitrary matrix $L$ to choose the basis gives

$$
\tilde{x}(t)=L \hat{Y}_{r}(t)=(L F) x(t)
$$

This shows that the new state vector will be a linear map of (28), so carrying out the update equations for $\tilde{x}$ will give us the same system, in a coordinate basis that corresponds to the similarity transformation $(L F)$.

\section{Consistency as the model order tends to infinity}

To investigate consistency - somewhat heuristically we shall assume that the number of data $N$, is so large that all estimates are effectively equal to their limiting values. In this section we shall also assume that the model order $s$ used in (7) is so large that the influence on $y(t+k)$ from input-output data older than $t-s$ is negligible. Alternatively, we may assume that the true system can be described by an ARX-model of order $s$. We shall also assume that the future input horizon $\ell$ in (7) is chosen so that $\ell \geq r$, so that all effects of inputs for $t+1 \leq i \leq k$ on $y(t+k)$ for $k$ up to $r$ are properly accounted for. All this means that (7) is a model structure that is capable of describing the $k$-step ahead predictors correctly. The estimates (10) will thus be the correct predictors.

For large $N$ and large $s$, the vector $\hat{Y}_{r}(t)$ will consequently have all the properties (24) - (29). The algorithm will therefore give the correct system description under these conditions. Note that this is true for all choices of $L$ that determine a basis for $\hat{Y}_{r}(t)$.

This approach can be seen as a variant of the two-stage methods described in Section 10.4 of [5]: First use a high order ARX-model to pick up the correct system dynamics (including noise dynamics), then reduce the model order by forcing the higher order model to fit a lower order one. In the Mayne-Firoozan method, [6] the innovations are used explicitly for this. In the subspace method, the predictions are used in a related way.

\section{Estimating only $A$ and $C$}

We can make the discussion in the previous section more focussed on the essential matters by concentrating on estimating $A$ and $C$. Once these matrices are fixed, estimating B and D in (1) is a linear regression problem, even for unknown $x$. (See (53), below.)

We then lump the dependencies of future inputs into a term $\tilde{\varphi}$ and set up a regression like

$$
\left(\begin{array}{c}
x(t+1) \\
y(t)
\end{array}\right)=\left(\begin{array}{l}
A \\
C
\end{array}\right) x(t)+\beta \tilde{\varphi}_{\ell+1}(t)
$$

If we use the $k$-step ahead predictors $\hat{y}(t+k \mid t)$ for the states $x$ as in (28), we then find - exactly as above that $A$ and $C$ will be consistently estimated if only the following three relationships hold:

$$
\begin{aligned}
\hat{y}(t+k \mid t)= & \hat{y}(t+k \mid t-1)+\beta_{1} \tilde{\varphi}_{\ell+1}(t) \\
& +\epsilon(t) \\
\hat{y}(t+n+1 \mid t)= & -a_{1} \hat{y}(t+n \mid t) \ldots-a_{n} \hat{y}(t+1 \mid t)+ \\
& +\beta_{2} \tilde{\varphi}_{\ell+1}(t)
\end{aligned}
$$




$$
\operatorname{rank}\left\{\hat{Y}_{n}(t+1)\right\}=n
$$

for some $\beta_{i}$ and a sequence $\epsilon(t)$ that is uncorrelated with $\tilde{\varphi}_{\ell+1}(t)$ and $\varphi_{s}(t)$. These relationships clearly hold for the true $k$-step ahead predictors as verified by $(24),(27)$ and $(6)$.

\section{Modifications to achieve consistency even for finite model orders}

The subspace methods can go one step further, and achieve consistent estimates of the $A, B, C$, and $D$ matrices, even without letting the model order $s$ tend to infinity. This is technically more involved. The basic idea is to establish that the key relations (31) and (32) will hold also for the approximate predictors $\hat{y}_{s, \ell}(t+k \mid t)$ and $\bar{y}_{s, \ell}(t+k \mid t)$ (defined by (10) and (11)), if only we play carefully with the "subscript orders" $s$ and $\ell$.

We start by establishing two lemmas for these quantities, which show properties analogous to Levinson type recursions.

Lemma 1 Suppose that the true system can be described by (25) with $n$ as the maximal order of the polynomials, and that the system operates in open loop, so that $e$ and $u$ are independent. Let $\hat{y}_{s, \ell}^{*}(t+k \mid t)$ and $\bar{y}_{s, \ell}^{*}(t+k \mid t)$ be the limits of (10) and (11) as $N \rightarrow \infty$. Then for any $s$, any $r>n$ and any $\ell \geq r$

$\hat{y}_{s, \ell}^{*}(t+r \mid t)+A_{1} \hat{y}_{s, \ell}^{*}(t+r-1 \mid t)+\ldots+A_{n} \hat{y}_{s, \ell}^{*}(t+r-n \mid t)=0$

and

$$
\begin{gathered}
\bar{y}_{s, \ell}^{*}(t+r \mid t)+A_{1} \bar{y}_{s, \ell}^{*}(t+r-1 \mid t)+\ldots+A_{n} \bar{y}_{s, \ell}^{*}(t+r-n) \\
=B_{0} u(t+r)+B_{1} u(t+r-1)+\ldots+B_{n} u(t+r-n)
\end{gathered}
$$

Proof: Consider the equation (7). Suppress the indices $\ell$ and $s$ and let

$$
\phi(t+1)=\left(\begin{array}{c}
\tilde{\varphi}_{\ell}(t+1) \\
\varphi_{s}(t+1)
\end{array}\right)
$$

Let $\zeta(t+1)$ be any vector of the same dimension as $\phi(t+1)$ such that

$$
\overline{\mathrm{E}} \zeta(t+1) C_{0}(q) e(t+r)=0
$$

Suppose that $\theta^{k}$ and $\gamma^{k}$ are estimated from (7) using the IV-method with instruments $\zeta(t+1)$. Then the limiting estimate are given by

$$
\left(\begin{array}{l}
\gamma^{k} \\
\theta^{k}
\end{array}\right)^{T}=\overline{\mathrm{E}} y(t+k) \zeta^{T}(t+1)\left[\overline{\mathrm{E}} \phi(t+1) \zeta^{T}(t+1)\right]^{-1}
$$

Note also that we can write, for some $\beta_{0}$

$$
B_{0}(q) u(t+r)=\beta_{0}^{T} \tilde{\varphi}_{\ell}(t+1)
$$

if $r>n$ and $\ell \geq r$, Hence

$$
\begin{aligned}
& \left(\begin{array}{c}
\gamma^{r} \\
\theta^{r}
\end{array}\right)^{T}+A_{1}\left(\begin{array}{c}
\gamma^{r-1} \\
\theta^{r-1}
\end{array}\right)^{T}+\ldots+A_{n}\left(\begin{array}{c}
\gamma^{r-n} \\
\theta^{r-n}
\end{array}\right)^{T}= \\
= & \overline{\mathrm{E}}\left[\left(A_{0}(q) y(t+r)\right) \zeta^{T}(t+1)\right]\left[\overline{\mathrm{E}} \phi(t+1) \zeta^{T}(t+1)\right]^{-1} \\
= & \overline{\mathrm{E}}\left[\left(B_{0}(q) u(t+r)+C_{0}(q) e(t+r)\right) \zeta^{T}(t+1)\right] \times \\
& \times\left[\overline{\mathrm{E}} \phi(t+1) \zeta^{T}(t+1)\right]^{-1} \\
= & \beta_{0}^{T}\left[\overline{\mathrm{E}} \tilde{\varphi}(t+1) \zeta^{T}(t+1)\right]\left(\begin{array}{c}
\overline{\mathrm{E}} \tilde{\varphi}(t+1) \zeta^{T}(t+1) \\
\overline{\mathrm{E}} \varphi(t+1) \zeta^{T}(t+1)
\end{array}\right)^{-1} \\
= & \beta_{0}^{T}\left(\begin{array}{ll}
I & 0
\end{array}\right)=\left(\begin{array}{ll}
\beta_{0}^{T} & 0
\end{array}\right)
\end{aligned}
$$

Here we used (39) and (37) in the third last step, and the definition of a matrix inverse in the second last step. Since

$$
\begin{aligned}
& \bar{y}_{s, \ell}^{*}(t+k \mid t)=\left(\begin{array}{c}
\gamma^{k} \\
\theta^{k}
\end{array}\right)^{T} \phi(t+1) \\
& \hat{y}_{s, \ell}^{*}(t+k \mid t)=\left(\begin{array}{c}
\gamma^{k} \\
\theta^{k}
\end{array}\right)^{T}\left(\begin{array}{c}
0 \\
\varphi(t+1)
\end{array}\right)
\end{aligned}
$$

we just need to multiply the above expression with $\phi(t+1)$ to obtain the stated result.

It now only remains to show that $\zeta(t+1)=\phi(t+1)$ obeys (37), so that the result holds for the least squares estimates. The vector $\phi(t+1)$ contains a number of inputs, which are uncorrelated with the noise, under open loop operation. It also contains $y(t)$ and older values of $y$, which are uncorrelated with $C_{0}(q) e(t+r)$ if $r>n$, since the order of $C_{0}$ is at most $n$. This concludes the proof.

Corollary: Suppose that the true system is given by

$$
A_{0}(q) y(t)=B_{0}(q) u(t)+v(t)
$$

and that the parameters of the predictors are estimated from (7) using an instrumental variable method with instruments $\zeta(t+1)$ that are uncorrelated with $v(t+r)$. Then the result of the lemma still holds.

Notice that the Lemma holds for any $s$, which could be smaller than $n$.

Lemma 2 Let $\bar{y}$ and $\hat{y}$ be defined as above. (These thus depend on $N$, but this subscript is suppressed.) Then for any $N, s, k$ and any $\ell$

$$
\begin{aligned}
y(t)= & \bar{y}_{s, \ell+1}(t \mid t-1)+\epsilon(t) \\
\bar{y}_{s+1, \ell}(t+k \mid t)= & \bar{y}_{s, \ell+1}(t+k \mid t-1)+\tilde{h}_{k, N}^{s+1, \ell} \epsilon(t) \\
\hat{y}_{s+1, \ell}(t+k \mid t)= & \hat{y}_{s, \ell+1}(t+k \mid t-1)+b_{k, N}^{s+1, \ell} u(t)+ \\
& +\beta_{k, N, s, \ell}^{T} \tilde{\varphi}_{\ell}(t+1)+\tilde{h}_{k, N}^{s+1, \ell} \epsilon(t)
\end{aligned}
$$

where $\epsilon(t)$ (same in the three expressions) is uncorrelated with $\varphi_{s}(t), u(t)$ and $\tilde{\varphi}_{\ell}(t+1), t=1, \ldots, N$. If the input sequence $\{u(t)\}$ is white, then

$$
b_{k, N}^{s+1, \ell} \rightarrow h_{u}(k) \text { and } \beta_{k, N, s, \ell} \rightarrow 0 \text { as } N \rightarrow \infty
$$


where $h_{u}(k)$ is the true impulse response coefficient.

\section{Proof: Let}

$$
\phi_{1}(t+1)=\left(\begin{array}{c}
\tilde{\varphi}_{\ell}(t+1) \\
\varphi_{s+1}(t+1)
\end{array}\right)
$$

and

$$
\phi_{2}(t+1)=\left(\begin{array}{c}
\tilde{\varphi}_{\ell+1}(t) \\
\varphi_{s}(t)
\end{array}\right)
$$

The vector $\phi_{2}(t+1)$ contains the values $u(i), i=t+$ $\ell, \ldots t-s$ and $y(i), i=t-1, \ldots, t-s$. The vector $\phi_{1}(t+1)$ contains the same values, and in addition $y(t)$. Define $\epsilon$ as the residuals from the least squares fit

$$
y(t)=L_{2} \phi_{2}(t+1)+\epsilon(t)
$$

so that $\epsilon(t) \perp \phi_{2}(t+1)$. With this we mean that

$$
\sum_{t=1}^{N} \epsilon(t) \phi_{2}(t+1)=0
$$

Note that $\epsilon(t)$ will depend on $\ell$ and $s$, but not on $k$. Moreover, by definition we find that

$$
L_{2} \phi_{2}(t+1)=\bar{y}_{s, \ell+1}(t \mid t-1)
$$

so the first result of the lemma has been proved.

Let

$$
\phi_{3}(t+1)=\left(\begin{array}{c}
\phi_{2}(t+1) \\
\epsilon(t)
\end{array}\right)
$$

It is clear that $\phi_{1}$ and $\phi_{3}$ span the same space, so that for some matrix $R$ (built up using $L_{2}$ ) we can write

$$
\phi_{1}(t+1)=R \phi_{3}(t+1)
$$

Now write

$$
y(t+k)=\hat{K}_{1} \phi_{1}(t+1)+\varepsilon(t+k)
$$

where $\hat{K}_{1}$ is the LS-estimate, so that

$$
\varepsilon(t+k) \perp \phi_{1}(t+1)
$$

Let

$$
\hat{K}_{1} R=\left(\begin{array}{ll}
K_{2} & K_{3}
\end{array}\right)
$$

Clearly, by definition

$$
\begin{aligned}
\bar{y}_{s+1, \ell}(t+k \mid t) & =\hat{K}_{1} \phi_{1}(t+1)=\hat{K}_{1} R \phi_{3}(t+1) \\
& =K_{2} \phi_{2}(t+1)+K_{3} \epsilon(t)
\end{aligned}
$$

Now rewrite (46) as

$$
\begin{aligned}
y(t+k) & =\hat{K}_{1} \phi_{1}(t+1)+\varepsilon(t+k) \\
& =\hat{K}_{1} R \phi_{3}(t+1)+\varepsilon(t+k) \\
& =K_{2} \phi_{2}(t+1)+K_{3} \epsilon(t)+\varepsilon(t+k)
\end{aligned}
$$

Both $\epsilon(t)$ and $\varepsilon(t+k)$ are orthogonal to $\phi_{2}(t+1)$, so $K_{2}$ must be the least squares fit of $y(t+k)$ to $\phi_{2}(t+1)$, which means that

$$
\bar{y}_{s, \ell+1}(t+k \mid t-1)=K_{2} \phi_{2}(t+1)
$$

Comparing (48) with (49) we have shown (42), with $\left.\tilde{h}_{k, N}^{s+1, \ell}=K_{3}\right)$. Moreover,

$$
\begin{aligned}
\bar{y}_{s+1, \ell}(t+k \mid t)= & \hat{y}_{s+1, \ell}(t+k \mid t)+\beta_{1} \tilde{\varphi}_{\ell}(t+1) \\
\bar{y}_{s, \ell+1}(t+k \mid t-1)= & \hat{y}_{s, \ell+1}(t+k \mid t-1)+\beta_{2} \tilde{\varphi}_{\ell+1}(t) \\
= & \hat{y}_{s, \ell+1}(t+k \mid t-1)+b_{k} u(t) \\
& +\beta_{3} \tilde{\varphi}_{\ell}(t+1)
\end{aligned}
$$

(with $b_{k}$ being the first column of $\beta_{2}$ ). Applying (42) to the two left hand sides of these expressions, we have also proved (43) (with $\beta_{k, N, s, \ell}^{T}=\beta_{2}-\beta_{3}$ and $b_{k, N}^{s+1, \ell}=$ $b_{k}$. The proof of (44) is straightforward and omitted here, since we will not need this result for the ensuing discussion. This concludes the proof of Lemma 2 .

\section{A consistent finite order Subspace Method}

Based on Lemmas 1 and 2, several different subspace methods can be derived that consistently estimate the $A, B, C$ and $D$ - matrices of a state-space model, even without letting the underlying model order tend to infinity. We here give just one such algorithm, which is a slight variant of the N4SID-method of [8]. It has the same first steps as the basic algorithm (12) of Section 7.5 .

\section{Algorithm SUBSP}

1. Estimate $\bar{Y}_{r}^{s+1, \ell}(t), \bar{Y}_{r}^{s, \ell+1}(t)$ and $\hat{Y}_{r}^{s, \ell+1}(t)$ using (16) and (17) with $\ell \geq r>\bar{n}$ where $\bar{n}$ is an upper bound of the system order.

2. Estimate the rank of $\hat{Y}_{r}^{s, \ell+1}$ and determine $L$ in (6), e.g. as in (19)-(23)

3. Introduce

$$
\begin{aligned}
\bar{x}(t+1) & =L \bar{Y}_{r}^{s+1, \ell}(t+1) \\
x(t) & =L \bar{Y}_{r}^{s, \ell+1}(t)
\end{aligned}
$$

4. Estimate $A, C$ and $\beta$ in

$$
\left(\begin{array}{c}
\bar{x}(t+1) \\
y(t)
\end{array}\right)=\left(\begin{array}{l}
A \\
C
\end{array}\right) x(t)+\beta \tilde{\varphi}_{\ell+1}(t)
$$

using the least squares method. (Notice the difference with (30). We here use "pseudostates" $x$ and $\bar{x}$ to make use of the subscript differences required to apply Lemma 2.) 
5. Keep the thus estimated $\hat{A}$ and $\hat{C}$-matrix fixed and estimate $B$ and $D$ in the linear regression

$$
y(t)=\left[\hat{C}(q I-\hat{A})^{-1} B+D\right] u(t)+\varepsilon(t)
$$

using the least squares method.

6. For the thus obtained $\hat{A}, \hat{B}, \hat{C}$ and $\hat{D}$, compute the residual covariance matrix $R$ by

$$
\begin{aligned}
& E(t)=\left(\begin{array}{c}
\bar{x}(t+1) \\
y(t)
\end{array}\right)-\left(\begin{array}{cc}
\hat{A} & \hat{B} \\
\hat{C} & \hat{D}
\end{array}\right)\left(\begin{array}{l}
x(t) \\
u(t)
\end{array}\right)(54) \\
& \hat{R}=\frac{1}{N} \sum_{t=1}^{N} E(t) E^{T}(t)
\end{aligned}
$$

7. If desired, compute from $\hat{A}, \hat{B}, \hat{C}, \hat{D}$ and $\hat{R}$ the Kalman gain $\hat{K}$ and the innovations representation

$$
\begin{aligned}
x(t+1) & =\hat{A} x(t)+\hat{B} u(t)+\hat{K} e(t) \\
y(t) & =\hat{C} x(t)+\hat{D} u(t)+e(t)
\end{aligned}
$$

Note that $s$ in (16) and (17) must be chosen large enough such that $x(t)$ in (51) has full rank.

\section{Verification of consistency of Algorithm SUBSP}

We assume throughout this section that the system operates in open loop so that the noise terms and the input are uncorrelated. This is in fact a necessary condition for consistency.

First note that for some matrices $V_{1}, V_{2}$ we have

$$
\begin{aligned}
\bar{Y}_{r}^{s+1, \ell}(t+1) & =\hat{Y}_{r}^{s+1, \ell}(t+1)+V_{1} \tilde{\varphi}_{\ell+1}(t) \\
\bar{Y}_{r}^{s, \ell+1}(t) & =\hat{Y}_{r}^{s, \ell+1}(t)+V_{2} \tilde{\varphi}_{\ell+1}(t)
\end{aligned}
$$

(see the end of the proof of Lemma 2). This means that the algorithm SUBSP will not be affected if we use $\hat{Y}$ instead of $\bar{Y}$ in (50)-(51). The estimates of $A$ and $C$ in (52) will be the same (although $\beta$ will of course be different).

To verify that SUBSP gives consistent estimates, let us consider the scalar output case and first assume that $L$ is chosen so that the first $n$ rows of $\bar{Y}$ are chosen as the states. Then we can word by word repeat the arguments in Sections 5 and 7 to find that the estimated $A$ and $C$ matrices are the correct ones and obtained in the observability canonical form. The ones and the zeros are obtained exactly also for finite $N$ in view of Lemma 2 , and the parameters of the last row will converge to the true ones in view of Lemma 1.

Once $A$ and $C$ are consistently estimated, the model (53) is a linear regression, with regressors entirely made up from the input sequence. The regressors are thus uncorrelated with any noise (we assume open loop operation) and hence also $B$ and $D$ will be estimated consistently.

Suppose now that a general matrix $L$ is chosen. If the true system is of order $n$, then $\hat{Y}_{r}$ is spanned by the $n$ first rows. If the states are formed from $\hat{Y}$, the the arguments applied to (29) holds without changes. If the states are formed from $\bar{Y}$ as in (50), then we will have that

$$
\tilde{x}(t)=(L F) x(t)+V_{3} \tilde{\varphi}_{\ell+1}(t)
$$

for some matrix $V_{3}$, instead of (29). That does not change the estimates of $A$ and $C$ (in the new basis).

Some further remarks: If the states are chosen as the first $n$ rows in $\bar{Y}$, then it follows from Lemma 1 that the last row of $\beta$ in (52) will converge to the true $B$ polynomial coefficients. With the $A$-parameters consistently estimated in the $A$-matrix, the $n+1$ first impulse response coefficients then can be correctly computed. These are the correct entries of the $D$ and $B$-matrices in this realization. With somewhat more effort these calculations can be carried out for an arbitrary choice of $L$. The step (53) is thus not necessary to consistently estimate $B$ and $D$, since this information is "hidden" in $\beta$ from the previous step.

It might be added that the covariance matrix $R$ in (54) will not be consistently estimated unless the correct $k$ step ahead predictors are used. For consistency of $K$ is is thus required that $s \rightarrow \infty$, in general.

If the states are chosen as linear combinations of $\hat{Y}$, and if the input is white noise, then it follows from Lemma 2 that $\beta \tilde{\varphi}_{\ell+1}(t)$ in (52) will converge to the correct term

$$
\left(\begin{array}{l}
B \\
D
\end{array}\right) u(t)
$$

In this case, $\tilde{\varphi}_{\ell+1}(t)$ can of course be replaced by $u(t)$, thus leading back to the basic algorithm (12).

\section{Conclusions}

We have in this contribution explicitly showed how the subspace identification algorithms are primarily based on estimates of the $k$-step ahead output predictors. We have also pointed to the main technical relationships (Lemmas 1 and 2) that are responsible for the consistency of the method, also in the case where too simple $k$-step ahead predictors are used. While the results as such are known from the previous literature, this analysis gives additional insights and might also help in developing an asymptotic second order analysis of these methods' statistical properties. 


\section{References}

[1] H. Akaike. Markovian representation of stochastic processes by canonical variables. SIAM Journal of Control and Optimization, 13:162-173, 1975.

[2] Larimore W. E. System identification, reduced order filtering and modeling via canonical variate analysis. In Proc. of the American Control Conference, San Francisco, CA, 1983.

[3] P. Faurre. Stochastic realization algorithms. In R. Mehra and D. Lainiotis, editors, System Identification: Advances and Case Studies. Academic Press, NY, 1976.

[4] T. Kailath. Linear Systems. Prentice-Hall, Englewood Cliffs, New Jersey, 1980.

[5] L. Ljung. System Identification: Theory for the User. Prentice-Hall, Englewood Cliffs, New Jersey, 1987.

[6] D. Q. Mayne and F. Firoozan. Linear identification of ARMA processes. Automatica, 18:461-466, 1982.

[7] J. Rissanen. Basis of invariants and canonical forms for linear dynamic systems. Automatica, 10:175182, 1974.

[8] P. Van Overschee. Subspace Identification, Theory - Implementation - Application. PhD thesis, Katholieke Universiteit Leuven, Kard. Mercierlaan 94, 3001 Leuven (Heverlee), Belgium, February 1995.

[9] P. Van Overschee and B. De Moor. Subspace algorithms for the stochastic identification problem. $A u$ tomatica, 29(3):649-660, 1993.

[10] P. Van Overschee and B. De Moor. N4SID: Subspace algorithms for the identification of combined deterministic-stochastic systems. Automatica, 30(1):75-93, 1994.

[11] P. Van Overschee and B. De Moor. Subspace Identification for Linear Systems - Theory Implementation Application. Kluwer, Dordrecht, The Netherlands, 1996.

[12] M. Verhaegen. Identification of the deterministic part of MIMO state space models given in innovations form from input-output data. Automatica, 30(1):61-74, 1994.

[13] M. Viberg, B. Ottersten, B. Wahlberg, and L. Ljung. Performance of subspace based state-space system identification methods. In Proc. 12th World Congress International Federation of Automatic Control, Sydney, Australia, volume 7, pages 369-372, 1993. 